This is the peer reviewed version of the following article: S. Ostermann, G. Kecskemeti and R. Prodan, "Multi-layered Simulations at the Heart of Workflow Enactment on Clouds," Concurrency and Computation: Practice and Experience, vol. 28, no. 11, pp. 3180-3201, August 2016, which has been published in final form at https://doi.org/10.1002/cpe.3733. This article may be used for non-commercial purposes in accordance with Wiley Terms and Conditions for Self-Archiving.

\title{
Multi-layered simulations at the heart of workflow enactment on Clouds
}

\author{
Simon Ostermann ${ }^{1 *}$, Gabor Kecskemeti ${ }^{2}$ and Radu $\operatorname{Prodan}^{1}$ \\ ${ }^{1}$ Institute of Computer Science, University of Innsbruck, 6020 Innsbruck, Technikerstr. 21a, Austria \\ ${ }^{2}$ Institute for Computer Science and Control, Hungarian Academy of Sciences, 1111 Budapest, Kende u. 13-17, \\ Hungary
}

\begin{abstract}
SUMMARY
Scientific workflow systems face new challenges when supporting Cloud computing, as the information on the state of the used infrastructures is much less detailed than before. Thus, organising virtual infrastructures in a way that not only supports the workflow execution, but also optimises it for several service level objectives (e.g., maximum energy consumption limit, cost, reliability, availability) become reliant on good Cloud modelling and prediction information. While simulators were successfully aiding research on such workflow management systems, the currently available Cloud related simulation toolkits suffer form several issues (e.g., scalability, narrow scope) that hinder their applicability. To address these issues, this article introduces techniques for unifying two existing simulation toolkits by first analysing the problems with the current simulators, and then by illustrating the problems faced by workflow systems. We use for this purpose the example of the ASKALON environment, a scientific workflow composition and execution tool for Cloud and Grid environments. We illustrate the advantages of a workflow system with directly integrated simulation back-end and how the unification of the selected simulators does not affect the overall workflow execution simulation performance. Copyright (c) 2015 John Wiley \& Sons, Ltd.
\end{abstract}

Received ...

KEY WORDS: Scientific workflow, Cloud computing, simulation toolkits

\section{INTRODUCTION}

Scientific workflows [1] enable constructing and executing large scale distributed applications based on well understood basic building blocks, designed for scientists with less expertise in organising and enacting a complex application. The burden of organisation and enactment lies on the underlying workflow management systems, that must not only ensure the proper and timely execution of the users' complex applications, but should also optimise their distribution and schedule on the available infrastructures. With the advent of Cloud computing [2], workflow

\footnotetext{
${ }^{*}$ Correspondence to: Institute of Computer Science, University of Innsbruck, 6020 Innsbruck, Technikerstr. 21a, Austria. E-mail: simon@dps.uibk.ac.at 
management systems must not only cope with the available infrastructures, but must also be able to decide when and how to improve user experience with the inclusion of leased virtual infrastructures.

Although the building blocks of these scientific workflow applications could have execution time in the range of months, their enaction by the workflow systems could have significant effects both on their runtime as well as on the underlying infrastructures [3]. In the past, several workflow systems used simulators [4,5] to evaluate the possible effects of particular enactment scenarios on workflows and infrastructures. Simulations are important tools to speed up research evaluations that otherwise would need too much time in reality. The increase in speed is normally reached by simplifying the model of the system to be simulated trying to stay as close to reality as possible. Simulations in some extreme cases are increasing evaluation speed to such levels that they allow close to real-time evaluation of multiple situations. Unfortunately, past workflow management techniques, which were incorporating simulators in their decision making process, hardly considered the highly volatile and dynamic nature of Cloud systems.

Although several Cloud simulators exist today (Cloudsim, GroudSim, iCanCloud) [6, 7, 8], they can hardly support the requirements of current workflow management systems. They are frequently oriented towards the Cloud user, therefore mostly considering Clouds as a black box. Unfortunately, this behaviour does not allow the analysis of infrastructure level effects of the various decisions made by workflow management systems. Even in such cases, when a simulator offers insights on how Clouds internally operate, they are mostly focused on specific areas (e.g. providing accurate CPU or network sharing, energy modelling) while neglecting others; therefore they restrict the use cases in which these simulators would be useful for the complex decision making process [9] in Cloud aware workflow management systems.

Through the example of a well researched scientific workflow management system (namely ASKALON [10]), we analyse in this paper the possible improvements one could gain by integrating a user-side simulator (called GroudSim [7]) with an internal infrastructure focused simulator (called DISSECT-CF, DIScrete event baSed Energy Consumption simulaTor for Clouds and Federations) [11]. Using this approach, we can not only fulfil the demands of current research directions, but also allow the widening of research applied in scientific workflow management systems. Thus, this paper has two distinct contributions: $(i)$ the integration of two complete simulators in a way that keeps their features while minimising the overhead caused by their joint operation, (ii) the analysis of new research directions the merged simulators could offer to the community researchers responsible for scientific workflow management systems.

We have chosen the ASKALON system because it has already been integrated with the GroudSim [7] simulator as a support tool in its workflow enactment-related decisions. For the role of the second simulator, we have selected the DISSECT-CF versatile simulation framework, as it is capable to simulate the internals of Cloud infrastructures allowing the evaluation of energy consumption, network behaviour and the effects of multi-tenancy. Although we have evaluated the integration on these specific systems, our carefully executed extensions show that the introduced techniques would be applicable to similar workflow systems too [12]. Our extensions show that an existing workflow system could already benefit from such integrated simulations with minimal or no changes to its workflow management techniques. The combination of ASKALON and the integrated GroudSim and DISSECT-CF simulators come along with many improvements which directly or 
indirectly influence the accuracy of the simulation results of scientific workflow applications in ASKALON.

The rest of the paper is organised as follows. In Section 2 we review the currently existing workflow systems and describe the advantages of our approach. In Section 3 we present the background information required to understand the existing systems in isolation, followed by details about the integration in Section 4. We summarise the new possibilities achieved by this extension in Section 5 and conclude the paper with a short outlook into upcoming work in Section 6.

\section{RELATED WORK}

Scientific applications are complex systems consisting of different programs that often need days or weeks to be executed. Users of such applications apply the workflow paradigm or other techniques to build larger scale applications out of existing programs, increase programming productivity and parallelism, and achieve faster execution times on. To research the impact of different schedules or optimisations, simulators are often employed to reduce the time between implementation of features and their verification.

The surveys in $[4,5]$ give a good overview of existing simulators, some of them covering the field of Cloud computing. The status of GroudSim in the [4] survey shows important missing features, while some features must have been overlooked by the authors (a cost model exists in GroudSim since its initial version, and has been extended over the years to support all commercially available billing models). Other crucial features provided by DISSECT-CF on the internals of infrastructure Clouds (e.g. energy models, more complex networking) are introduced in this publication and have been added to GroudSim too.

GridSim [13] and its extension CloudSim [6] are well-known simulation environments for task executions on Grid and Cloud platforms. As our previous work showed, the scalability and flexibility is their biggest problem [7].

iCanCloud [8] is a new contribution to the area of Cloud simulators specialised on Amazon EC2 resources using a configuration GUI. Because of its user orientation, iCanCloud lacks crucial functionality needed for Green IT research [14] such as power consumption, and has no workflow support.

WorkflowSim [15] is an open source workflow simulator that extends CloudSim by providing new constructs for simple management and simulation of workflows. It models workflows as a DAG and provides out of the box implementation for several popular workflow schedulers (e.g., HEFT, Min-Min) and task clustering algorithms. Its main disadvantage (alongside its limitation to the DAG model lacking loops) is that it misses a connection to a real-life workflow management system such as ASKALON.

SimGrid [16] has been developed over the past years as a versatile, accurate and scalable simulator. Compared to our solution, it lacks support for dynamic workflow applications, as it only supports static DAGs, and does not offer important features like real-life and simulated executions within the same environment. Researching new methods and ideas needs therefore twice effort required in ASKALON: first the validation must be performed in SimGrid, and afterwards the new code needs to be rewritten for the real execution environment. 
The previews integration work of GroudSim into ASKALON [17] showed the usefulness of such a integrated approach that other workflow systems such as Pegasus [18], Taverna [19] or WS-PGRADE [20] lack. With the integration of DISSECT-CF, all features added to GroudSim are also automatic available in ASKALON, allowing better simulations leading to more accurate and realistic research results.

Compared to other existing simulators, two features make the combination of the ASKALONGroudSim system with DISSECT-CF unique: $(i)$ the possibility to simulate and execute workflow applications directly within the same environment, and (ii) the integration of a unified power utilisation and resource sharing model for simulating data centre components.

\section{BACKGROUND}

Simulation is a known useful practice when trying to solve complex problems like scheduling, resource management or workflow executions. There are multiple tools available for this purpose, as mentioned in section 2, but they either lack functionality or are not user friendly. Especially the high interest in power-aware methods is not satisfactory with the current available simulators in the scope of workflow executions on Cloud resources. To overcome this drawback, we developed a simulator specialised on Infrastructure as a Service (IaaS) Clouds with focus on power consumption and scalability of the simulation. We integrated this simulator into an existing framework for workflow development, execution and simulation called ASKALON.

\subsection{ASKALON}

ASKALON, an existing middleware researched at the University of Innsbruck, provides an integrated environment to support the development, simulation and execution of scientific workflows on dynamic Grid and Cloud infrastructures [10]. Figure 1 shows the design of the ASKALON system with focus on the integrated simulator, explained in detail in Section 3.2. Workflows can be graphically programmed in an abstract and user-friendly fashion in a platform independent Java application. The abstraction is used to shields the users from the low-level Cloud infrastructure technology details as no such knowledge is needed in the workflow creation process. Workflows can be created in a "drap and drop" fashion form existing abstract activities where only the input and output port must be connected to each other to build the workflow structure. Once the workflow is created and confirms to the model checker, it can be submitted for execution to the ASKALON services, which allow for long lasting executions in online interactive or offline batch mode. A command line client allows script-based batch execution of the workflows in an XML language representation for cron-job based executions of single or multiple workflows. Execution information is stored in a database allowing online and post-mortem analysis using a graphical performance measurement tool or custom SQL-queries. The three main components that handle the execution of workflows are explained in the following.

Execution Engine. The execution engine (EE) is responsible for processing the workflow, unrolling the parallel loops into executable tasks and their management. Submission of jobs and transfer of data to the compute resources is done with a suitable protocol such as ssh 
and scp for Cloud resources or GRAM and GRIDFTP in a Globus/Grid environment. For simulated workflow executions, we developed a new provider for the Globus CoG-kit [21] that allows the use of the existing abstraction model to interact with the integrated simulator. There is also a scheduling module included in the EE2 that allows an easy integration of existing and new scheduling algorithms (e.g. MOHEFT [22], HEFT [23], MCT [24]).

GridARM/GLARE. The resource manager has the task to manage existing Grid resources or to provision the correct amount of Cloud resources at the correct moment to allow the EE run the workflow as decided by the scheduler. The scheduler can therefore include precise information in the task mappings (i.e. run the task on a specific resource from a Cloud provider) or less restrictive mappings (i.e. run a task on any resource of Cloud provider). To request and release Cloud instances, the resource manager communicates with different Cloud providers, or in the simulation case with GroudSim, to provision Cloud instances using predefined images for the required applications. The applications may be automatically deployed on the instance after its boot or the image may already include the desired applications.

GAB. The GroudSim-ASKALON-Bridge is responsible for distinguishing between the components of ASKALON that can only be effectively run in real a real environment, and the simulated execution environment operated by GroudSim. As ASKALON is used for executions on real hardware, this module is needed to allow the integration of the simulator in a transparent fashion to the other components that are not simulated such as the scheduler, resource manager and job or file transfer submissions. This module ensures that the simulation time is only advanced when no more new events from ASKALON are generated to avoid increased simulated time due delayed job submissions. As the EE is heavily using threads for pipelining and parallelism in the processing the workflow structure and execution, we aim not to stop it more then needed. Therefore, most EE threads continue working while other functions are blocked using advanced lightweight Java synchronization mechanisms (BlockingQueues), as they have to wait for the simulated results. This enables a better performance of the system, but does not allow to clearly identify the overhead added with this synchronization mechanism as most threads are not blocked. Adding blocking mechanisms to all EE threads would allow to better measure GAB overheads but this would significantly decrease the overall performance meaning that EE threads need to support pause functions. Therefore, we decided our design for performance in order not to add additional overheads for making this mechanism better measurable.

\subsection{GroudSim}

GroudSim is an event-based simulation toolkit for scientific applications running on combined Grid and Cloud infrastructures developed in $\mathrm{Java}^{\dagger}$. GroudSim uses a discrete-event simulation toolkit that consists of a future event list and a time advance algorithm that offers improved performance and scalability compared to other process-based approaches used in related work [25]. The simulator can be used in a stand-alone fashion or integrated in the ASKALON environment. The later

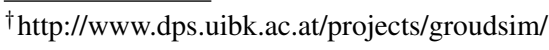




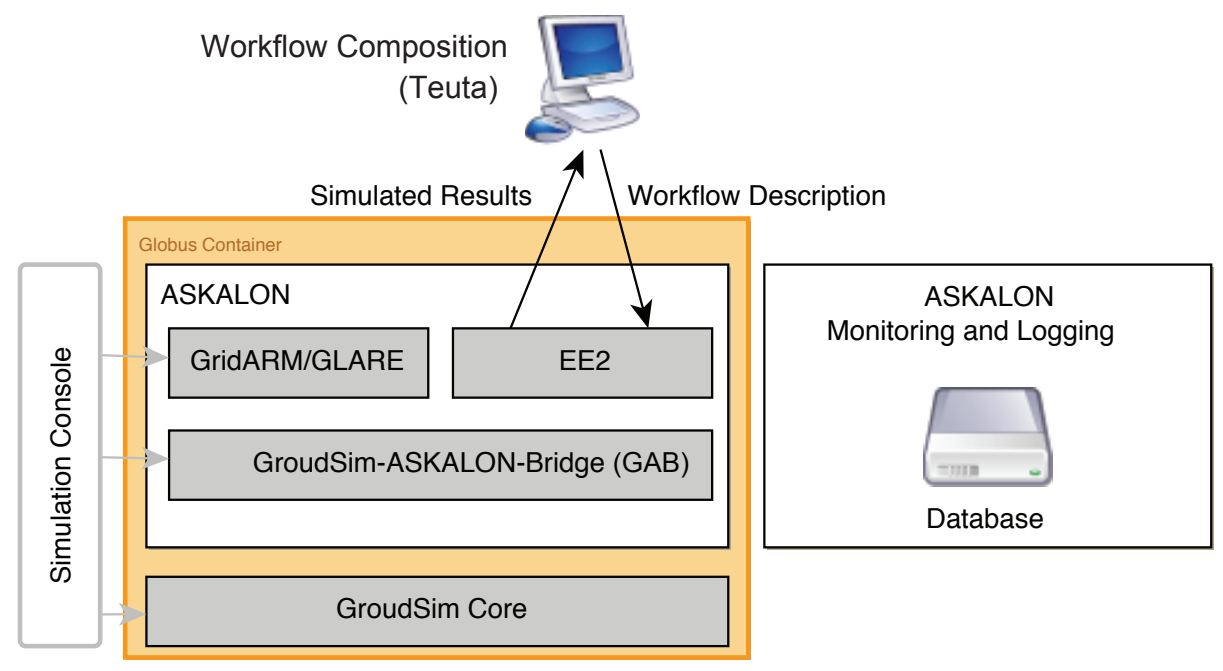

Figure 1. The architecture of ASKALON

option allows seamless development, debugging, simulation and execution processes using the same ASKALON interface offered to the end-users.

Figure 2 shows the most important components of GroudSim, which collaborate internally and act as interfaces to communicate with the GAB. The two central parts of the simulation framework are: (i) the event system storing information about the type and time of the events, and (ii) the simulation engine responsible triggering the events at well-defined time instances. Events can simulate job executions, file transfers, availability of resources (including failures), and background load. The other GroudSim core components are:

Resource module that manages the simulated resources and communicates them to GridARM/GLARE;

Synchronisation module which allows synchronisation of the simulation time and the time used by the EE. When the EE is generating new tasks and submits them to the simulator, the simulator must wait until all current tasks are submitted before the simulation time can be advanced;

Background loader adds additional load to the resources upon requests from the user or GAB. The load can be achieved by using traces from the Grid Workload Archive [26] or by using synthetic job distribution functions;

Failure generator which handles the failure rates for jobs, file transfers and resources following stochastic distributions;

Stochastic framework that offers different stochastic distribution functions, which can be used for calculating queuing times, submission times, execution times, failure rates or background loads;

Tracing module which is used to store the simulated execution events to a file for analysis or debugging. 


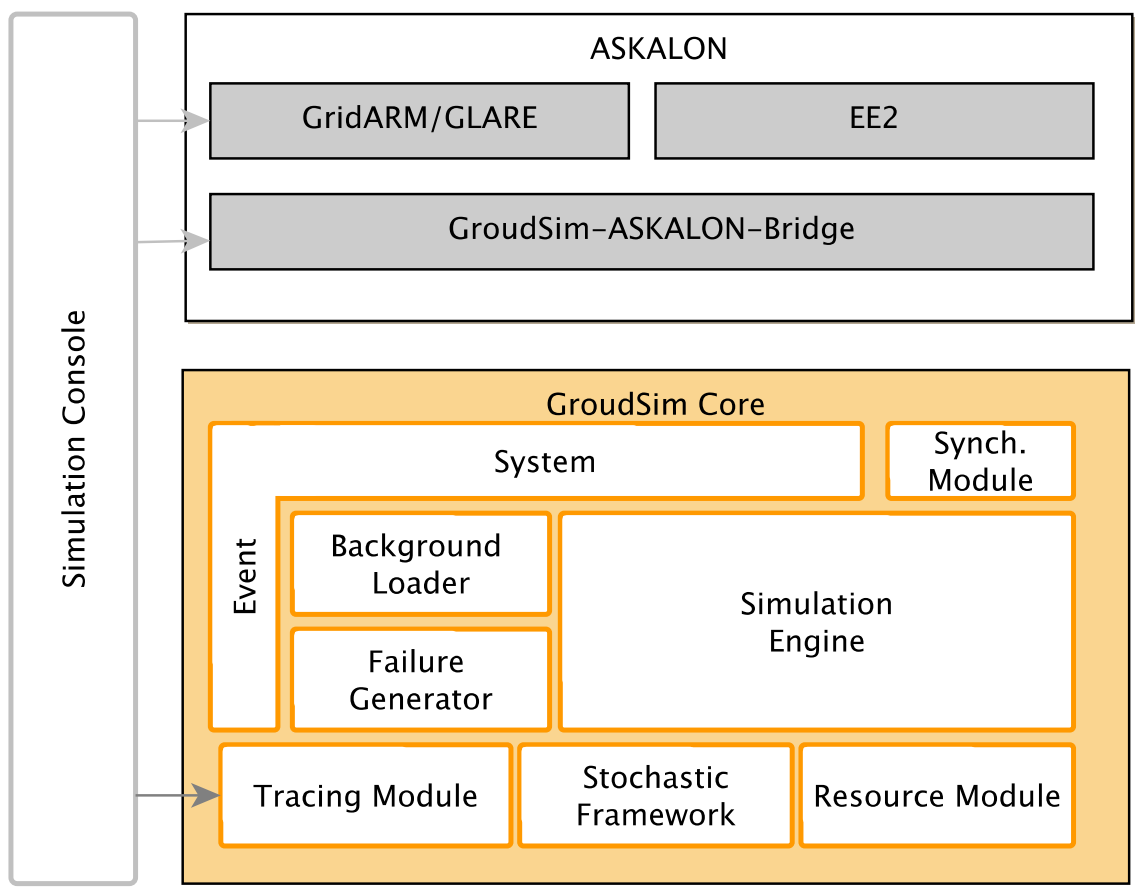

Figure 2. The architecture of GroudSim

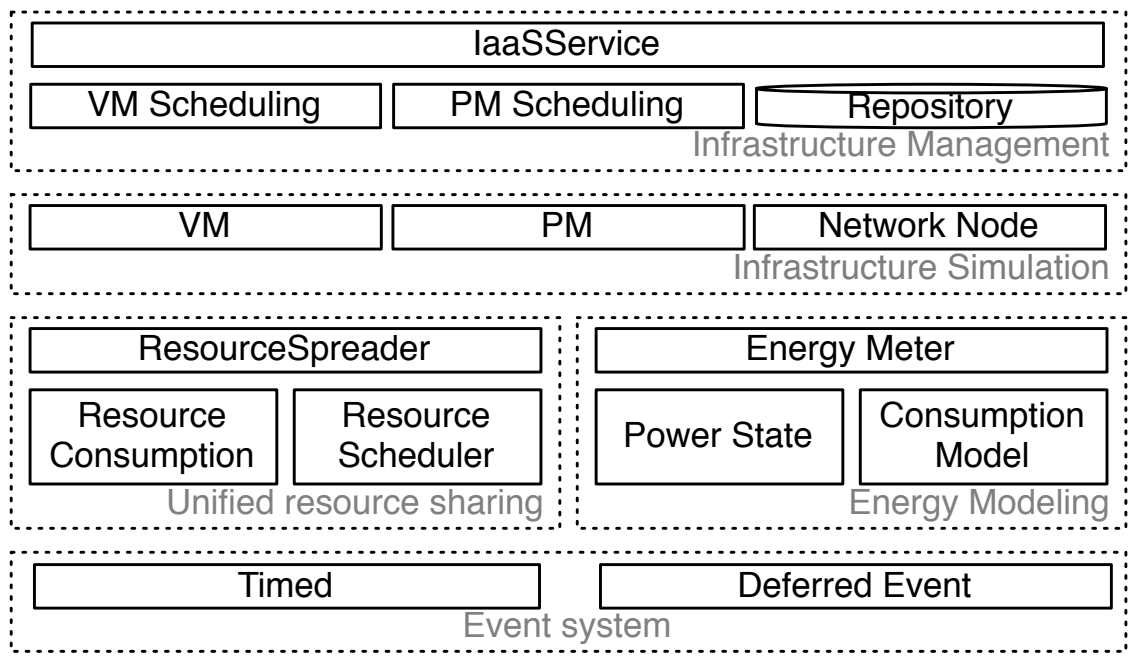

Figure 3. The architecture of DISSECT-CF

In addition to these services, the GroudSim enabled execution and simulation service provides a GUI that allows easy setup of the Grid and Cloud resources by showing statistical charts of the simulated tasks and file transfers. All this information can also be collected in the performance database or setup via configuration files, if preferred.

\subsection{DISSECT-CF}

As we plan to support future research in the ASKALON workflow enactment, we aimed at increasing the capabilities of GroudSim with the least effort. Unfortunately, GroudSim's lack of 
internal IaaS behavioural knowledge reduces the number of future use cases that could be supported in the ASKALON-GroudSim system. Therefore, we have analysed several simulators that could act as the foundation for GroudSim and offer insights about the internals of IaaSs. Since GroudSim's focus was primarily on performance and efficiency, we selected the DISSECT-CF simulator to complement its functionality that has a good performance, while having similar internal concepts of time, events, and infrastructure

DISSECT-CF [11] is a compact, highly customisable open source Cloud simulator with special focus on the internal organisation and behaviour of IaaS systems. Figure 3 presents the architecture of the currently available $\mathrm{e}^{\ddagger} 0.9 .5$ version. The figure groups the major components into subsystems marked by dashed lines. Each subsystem is implemented as independently from the others as possible. There are five major subsystems, each responsible for a particular aspect of the internal IaaS functionality: $(i)$ event system for a unified time reference; $(i i)$ unified resource sharing to resolve low level resource bottleneck situations; (iii) energy modelling for the analysis of energy usage patterns of individual resources (e.g., network links, CPUs) or their aggregations; (iv) infrastructure simulation to model physical and Virtual Machines (VMs) as well as networked entities; and finally $(v)$ infrastructure management to provide a real-life Cloud API and encapsulate Cloud-level scheduling.

After the simulators are integrated, the new ASKALON workflow enactors can perform better by utilising more information than the previously available job run-times and VM execution prices. Thanks to DISSECT-CF, the new enactors will be capable to use VM instantiation timings, job/VM or even workflow level energy consumption details and a more precise network and CPU process model. On the other hand, DISSECT-CF, if used through GroudSim, will immediately gain Cloud pricing capabilities and the possibility to involve hybrid workloads by utilising both Clouds and Grids in a single simulation. The following section details how the integration of the two simulators enables these new functionalities.

\section{INTEGRATION}

Throughout the integration, we have aimed at maintaining API compatibility of GroudSim, thus ensuring that past work on GAB does not need to be repeated. We have investigated the APIs of both GroudSim and DISSECT-CF and we have analysed the bridging functionalities needed to cross simulator boundaries. According to our analysis, there are three major areas where the simulators have significantly differing but relevant APIs for our goals to enable more sophisticated simulation based workflow enactment. These three areas are the following: $(i)$ the event systems have different event types, event firing mechanisms, clock maintenance techniques; (ii) Cloud representations have conceptual disagreements on data centre organisation, VM and job management mechanisms; and finally (iii) network construction, utilisation, sharing and organisation. The rest of this section discusses how the gaps amongst these areas were closed allowing us a seamless transition from GroudSim level simulation to the abstraction used in DISSECT-CF.

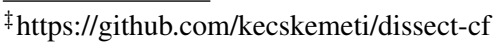




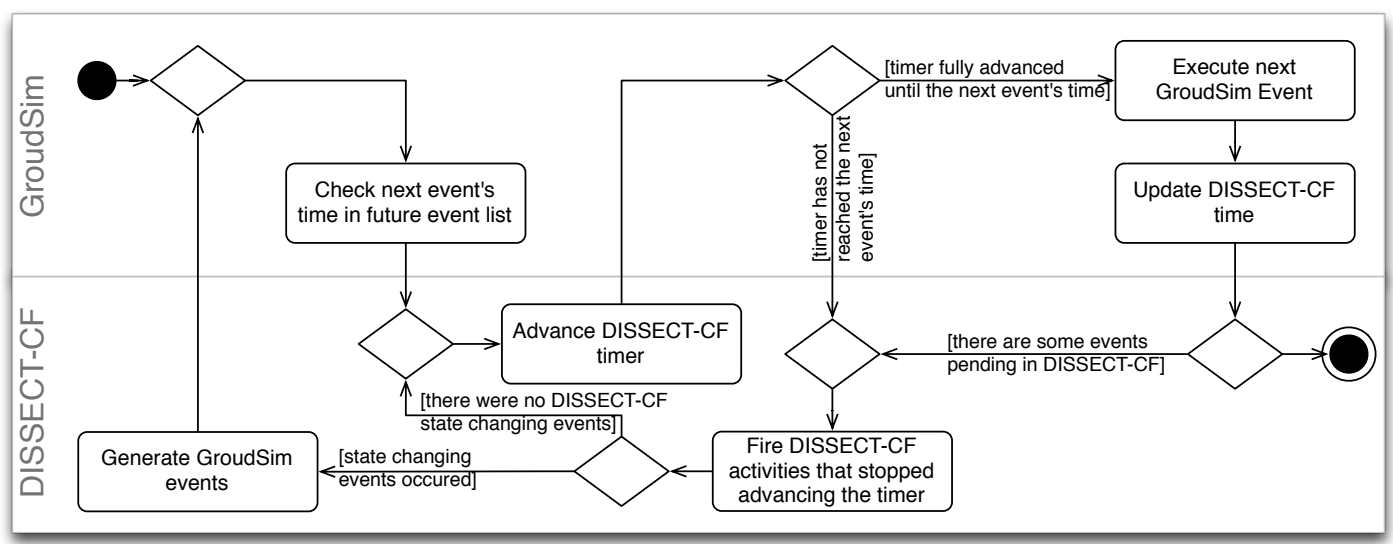

Figure 4. Interaction between GroudSim and DISSECT-CF within the main event processing loop of GroudSim's simulation engine. Remark: the diagram only captures the processing of a single GroudSim event within the loop

\subsection{Event systems}

First of all, we have chosen to make GroudSim as the master simulator. As a result, there should not be events in DISSECT-CF unless there was a preceding GroudSim event that caused a series of DISSECT-CF ones. Second, if some activity happens in DISSECT-CF that has an equivalent in GroudSim, then it must be ensured, that DISSECT-CF level events are never sent directly to the user of GroudSim. Instead, they must set off an equivalent GroudSim event (see Figure 4's GroudSim event generation activity). This technique ensures, that simulations utilising GroudSim features never need to be aware of the internals of the DISSECT-CF based activities, while the technique also reduces the number of events that must go through GroudSim and thus increases the performance of the integrated simulators.

Now that we have seen how events could occur cross simulation boundaries, let us focus our attention on the way the timing of these events are also managed in both simulators simultaneously. To keep the two simulators synchronised, we have chosen to extend the simulation engine of GroudSim. This extension alters the simulators future event list processing and inside its event loop it always ensures that at any given time instance neither DISSECT-CF nor GroudSim has events, which should have happened already according to the maintained time in the other simulator. The new extension is depicted in Figure 4. The time of GroudSim is kept in sync with DISSECT-CF by ensuring that only GroudSim's simulation engine controls the time of the underlying DISSECT$\mathrm{CF}$ simulation (see the time advancement and update activities in the Figure). The extension also handles situations when events in one of the simulators cause events in the other one (e.g., see the last conditional activity on the side of GroudSim in the Figure). This is especially important as DISSECT-CF has two kinds of events: time- and state dependent ones. Time dependent events are placed in the event queue of the Timed class, but state dependent events are fired by the entities that have had their states observed. In GroudSim, these two kinds of events are linked with the technique of event references and during their creation every event has its occurrence time predetermined. However, to reduce the synchronisation overhead, we choose not to create event references in sync with GroudSim (as the occurrence times are not yet available for state dependent events). Instead, 
when a state dependent event occurs, we request GroudSim to insert a new event into its queue for immediate execution (see the end of the DISSECT-CF activities in Figure 4).

\subsection{Cloud representation}

Originally, infrastructure Clouds were conceptually differently simulated in the two simulators. While GroudSim focused mostly on the blackbox Cloud model, DISSECT-CF offered insights on the internals of IaaSs. The blackbox model allowed GroudSim to abstract away such activities like VM creation details, VM placement and physical machine state scheduling. This model ensured the performant evaluation of Cloud related workloads. Unfortunately, the blackbox model cannot be applied successfully to Cloud infrastructures with limited resource capabilities such as private or academic Clouds because the abstracted activities could make significant differences to the outcomes of VM operations. Thus, we choose to keep the APIs of GroudSim, but dropped the blackbox model and ensured that DISSECT-CF simulates the previously abstracted functionalities. Although, this addition introduces some performance penalties, we have chosen DISSECT-CF because it has been shown to be a better performer than other simulators with similar features.

4.2.1. Cloud infrastructure management Because of GroudSim's blackbox approach, Clouds are defined by two properties: the number of cpu cores and the set of VM instance types one can create on top of the Cloud. On the other hand, in DISSECT-CF, one can define the kinds and the amounts of physical machines that constitute the Cloud, and it is also possible to set energy consumption properties, custom VM and physical machine schedulers. The integrated version introduces more flexibility to GroudSim's Cloud representation on the following two approaches: (i) limited customisability restricted to the number and kind of physical machines; and (ii) extended customisability that enables better energy awareness through customisable consumption properties and physical machine schedulers. Unfortunately, even with the extended approach the customisation of VM schedulers is not entirely possible because GroudSim expects Clouds to reject VM instance requests that cannot be served in the current state of the simulated IaaS. This behaviour is similar to what one can expect from commercial Cloud systems and several academic Cloud wares (like OpenNebula) currently. Thus, it is supporting the research on such IaaSs that are available today. The evaluation of workflows in future IaaS constructs is not supported without conceptual changes in GroudSim's Cloud representation.

Next, we are going to detail the approach of limited customisability. In this case, the user of GroudSim is not expected to know that at the background there is another simulator for the internals

of Cloud infrastructures. In such case, we expect that users first define what kind of instances they will need from a particular Cloud. Our approach then determines the maximum number of CPUs, the top performance (in terms of MIPS/core), and the biggest amount of memory needed by any of the user defined instances. These maximums are used for the definition of the template physical machine which will be the foundation of the DISSECT-CF Cloud infrastructure. In DISSECT$\mathrm{CF}$, we will create as many of these kind of physical machines as many can match the amount of CPU cores asked by the user for the particular Cloud during its construction. The created physical machines will all be connected together via a Cloud level network and the internal DISSECT-CF Cloud representation will also simulate a single repository to store a single kind of virtual appliance from which all the VMs can be derived. The physical machines will be controlled by a physical 


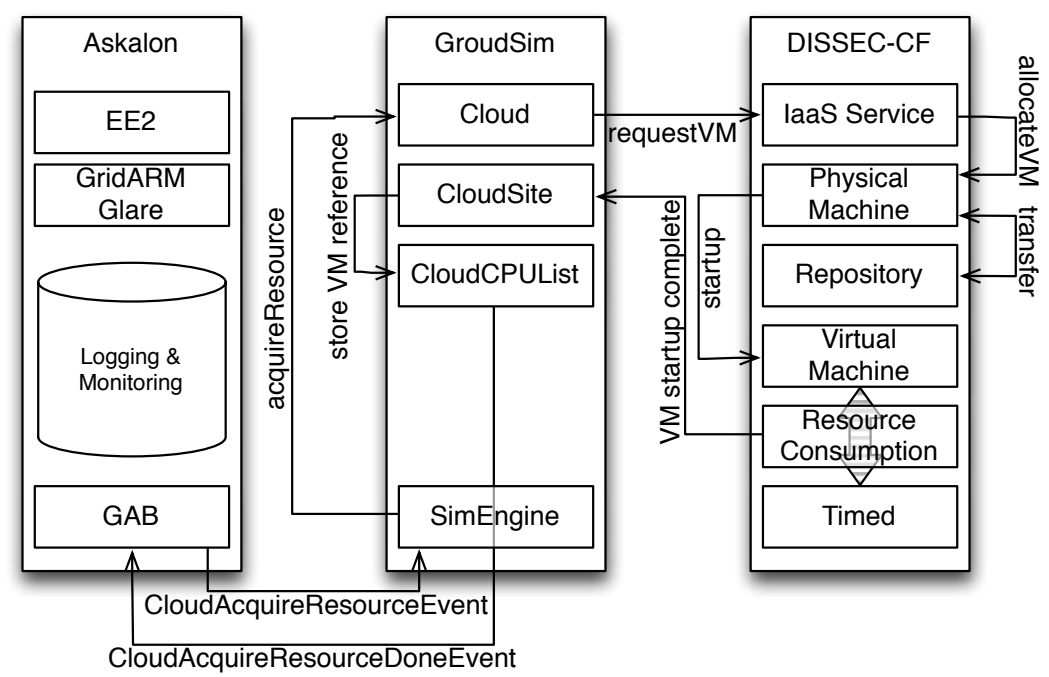

Figure 5. Extended VM instantiation procedure

machine scheduler that keeps them always on. As it can be seen, this infrastructure is rather limited and as a result it also seriously limits the possible evaluation scenarios the integrated simulators can support.

To remove some of these limitations, the simulator also allows the loading of the internal Cloud's properties via a file. In this file, users can define the topology of the physical machines, also they can provide custom power profiles to them and finally they can change the physical machine schedulers as well. These alterations enable network and energy aware workflow enactment, but demand user knowledge about the creation of the Cloud description file that DISSECT-CF can process with its CloudLoader. Fortunately, the Cloud description file allows us to keep the GroudSim APIs unchanged and to alter IaaS behaviour from one simulation run to another by just changing these descriptors.

4.2.2. Binding between the two VM representations DISSECT-CF allows flexible and continuous resource constraint control during its VM instance creation mechanism (i.e., users can ask for arbitrary cpu, memory and processing capabilities for their future VMs). This is similar to the behaviour of several academic Cloud wares. Unfortunately, the instance type system of GroudSim significantly limits the possible kinds of VM instances one can create similarly to how Amazon EC2 limits their users. To keep the Cloud concept of GroudSim, in the integrated simulation, we have limited the continuous resource constraint space of DISSECT-CF to the instance types from GroudSim.

To handle Grids and Clouds uniformly, GroudSim considers a single VM as a CloudSite. Therefore, such resources are scheduled by OS level schedulers instead of local resource management systems applied in Grid systems. In GroudSim, CloudSites are requested with an instance type. As depicted by Figure 5, this request is then forwarded to DISSECT-CF. Where the simulation schedules the VM to the most suitable physical machine. If the current physical machines in the Cloud are too loaded and cannot serve the requested instance type, the VM scheduler will mark the requested VM as non-servable, allowing DISSECT-CF to fail the CloudSite 
acquisition process in GroudSim. If the VM request can be allocated to a physical machine, the VM is instantiated on it (by simulating the transfer of its virtual appliance to the necessary storage element and then by simulating its startup procedures). Finally, the GroudSim user is notified about the creation of the new VM.

It must be noted, that in GroudSim, one cannot provide any relevant information to differentiate the planned function of newly created VMs. As a result, currently GroudSim always instantiates DISSECT-CF VMs with the same virtual appliance. As appliance size is a significant factor in VM creation time, this loss of differentiation between virtual appliances reduces the variance of VM creation times significantly. Therefore, even in DISSEC-CF enhanced GroudSim simulations, the variance of a particular appliance's transfer can be affected only by network activities like transfers between VMs or significant VM creation bursts. Later on, we will further extend GAB so it will be able to forward the expected functionality of a future VM by sending the properties of the applications planned to be run on the VM under creation.

4.2.3. Job scheduling Since GroudSim did no mapping between physical and VMs, there was no chance to observe several phenomena that occurs in under-provisioned Clouds. CloudSites processed jobs independently from other CloudSites in the particular Cloud infrastructure, despite they could share resources in the background. This sharing reduces the accuracy of GroudSim in scenarios involving heavy Cloud usage.

Jobs in GroudSim are also restricted to use a single cpu core. In Grid sites this restriction is further extended so one CPU is not allowed to have multiple jobs. But GroudSim removes this restriction for Clouds. As a result, GroudSim allows the simulation of simple VM level resource bottlenecks. Unfortunately, this bottleneck situation is less frequent in Clouds, especially with job models when one cannot reduce or suspend the processing of a job if needed (e.g., because of changing application characteristics or job migration across VMs).

The above mentioned issues hinder the evaluation of advanced scheduling and workflow enactment techniques applied in ASKALON. Thus, during the integration, we have aimed at removing these limitations. With its unified resource sharing mechanism, DISSECT-CF offers a widely applicable resource scheduling technique that can efficiently and more accurately manage resource bottleneck situations.

After the integration, a GroudSim simulates a job in two phases. First, GroudSim manages the job's lifecycle until it should be running on a CloudSite. In that case, the selected CloudSite injects a new CPU level resource consumption into the VM representing the site in DISSECT-CF. Then comes the second phase of job execution: DISSECT-CF applies its resource sharing technique that automatically considers both the physical machine's load, which hosts the VM, and also the currently processed jobs in the VM. If a simulation set up a Cloud infrastructure with a VM scheduler that allows under-provisioned VMs, then the jobs will experience performance drops automatically. Also, the integrated simulators allow jobs to have limited performance for some periods of time and cancellation free job migration across other DISSECT-CF simulated VMs.

\subsection{Networking}

GroudSim have offered customisable network links among both Grid- and Cloud sites. These links were connected to GroudSim's central bus representing the Internet (see Figure 6). FileTransfers 


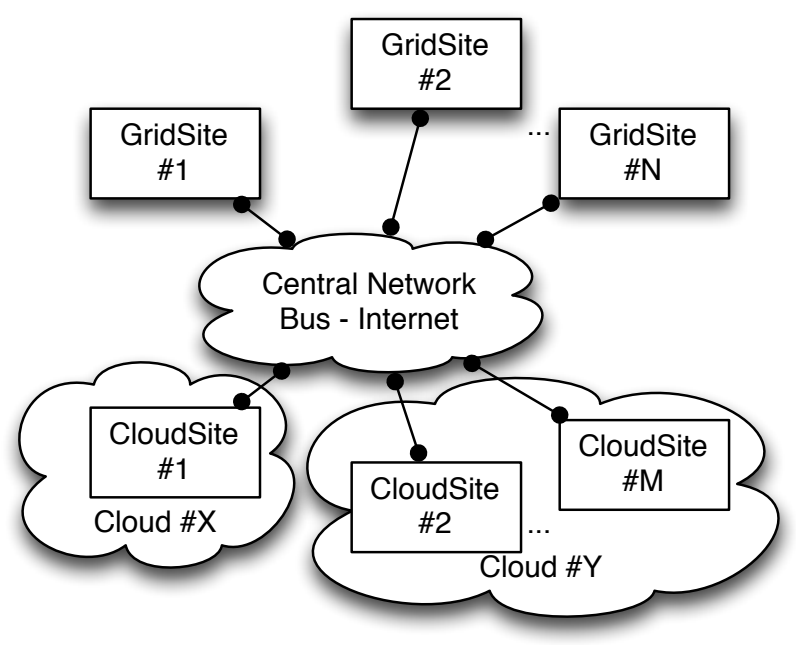

Figure 6. Original GroudSim network topology

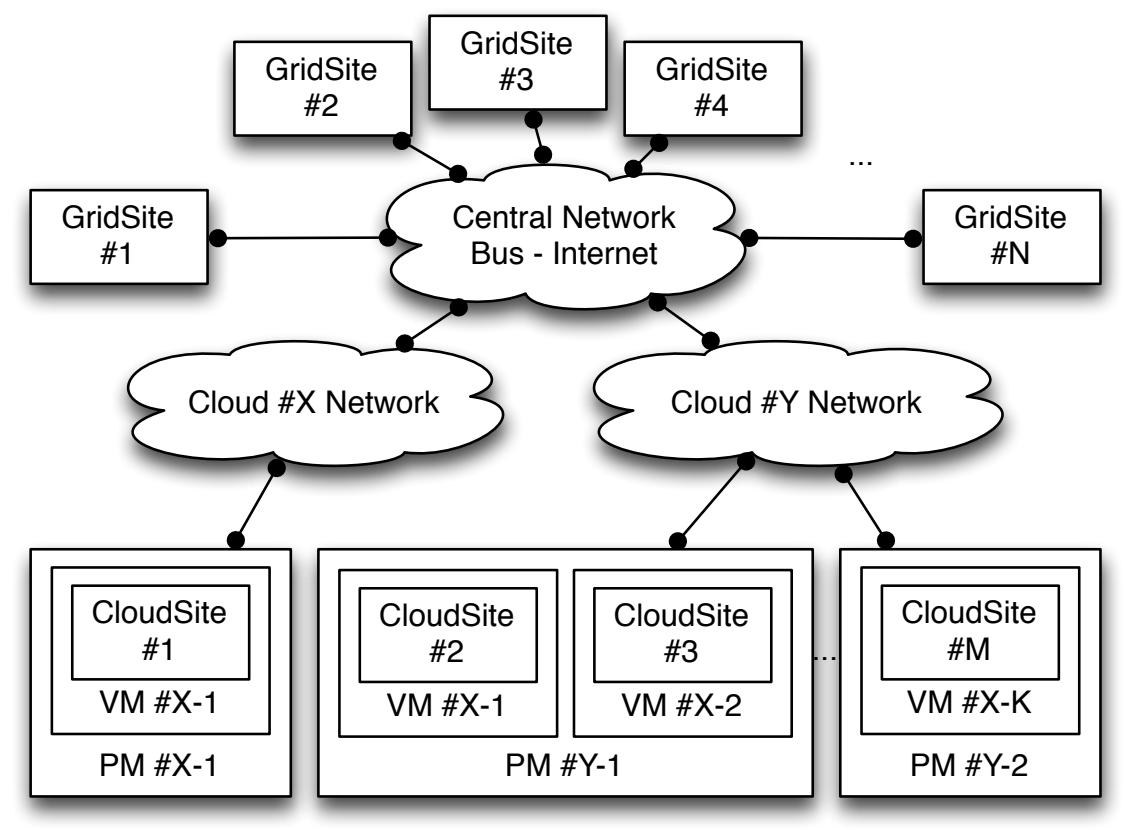

Figure 7. Integrated GroudSim-DISSECT-CF networking

between two sites then passed through two network links and the Internet. When bandwidth was utilised by multiple file transfers, the share of each transfer was estimated based on the network link with the smallest bandwidth. This estimate, however, often lead to unused network capacities and highly inaccurate network bandwidth utilisation compared to real life or packet level simulator results.

Although, DISSECT-CF still offers a simplified network model, it can model GroudSim's central bus topology without any modifications. Also, thanks to its unified resource sharing model, it can immediately offer a solution to network resource bottleneck resolution. Clouds loaded with extended customisability can even limit their overall connections to GroudSim's central bus as a whole. As 
a result, DISSECT-CF extended simulations can organise GroudSim's Clouds and Grids into a hierarchical network (see Figure 7) where new workflow enactment techniques could investigate the effects of alternative Cloud deployment layouts on network transfers, latencies and VM instantiation times.

\section{IMPROVED ASKALON BEHAVIOUR}

In this section we will present scalability experiments based on simulation of real world workload traces simulated with the original GroudSim and the merged version to show that the added functionality did not result in significant simulation performance degradation. Then, we highlight new possibilities in the workflow system made possible by the DISSECT-CF features. A detailed subsection about the simulations within ASKALON show how the merged simulators improve workflow development and utilisation. Finally, the section discusses how the composite ASKALON-GroudSim-DISSECT-CF system scales during the simulated execution of a workflow.

\subsection{Scalability experiments}

After the integration of DISSECT-CF into GroudSim, we first aimed at determining the performance and scalability penalties introduced into GroudSim because of the additional features available through DISSECT-CF. We expected a performance drop because of the cross-simulator time synchronisation and the more detailed infrastructure simulation techniques. In order to evaluate the properties of the integration, we have chosen several simulation scenarios: $(i)$ simulating realistic background loads with the help of GroudSim's background loader and the Grid Workload Archives (GWA); (ii) evaluating the improved networking capabilities via simultaneous network transfers; and (iii) evaluating the simultaneous VM instantiation performance of the simulators to show their applicability in large-scale environments. In all three cases the evaluation was done in three phases: (a) implementing the intended simulation in both the new and the old versions of GroudSim; $(b)$ validating the new version of GroudSim by comparing its simulation results to the ones received from the old version - all validating runs were within 0.01 ; and finally $(c)$ once the two simulations were considered equivalent we have evaluated how long they take to perform the selected evaluation scenarios. In the last phase, each scenario was ran in several (later detailed) setups by both the new and the old GroudSim. For each run we collected a time that took to run the setup $-t_{\text {set }}$. Performance evaluations of a particular setup were executed until the sample standard deviation $-s_{N}-$ of the $t_{\text {set }}$ values becomes stable, which means that the value of two ensuing standard deviation calculations are within $1 \%$.

$$
\frac{s_{N}\left(t_{s e t}\right)-s_{N+1}\left(t_{s e t}\right)}{s_{N}\left(t_{s e t}\right)}<0.01 \text { where } N \geq 2
$$

In practice, for the below detailed setups, this requirement resulted between 7-22 measurements. When a figure presents $t_{\text {set }}$ values it is always presenting the median of the values obtained for the sample standard deviation calculations.

Evaluation through GWA traces. We have selected the GWA as the base for the workload because GroudSim already has a loader - called Background Loader in Fig. 2 - for it and its 


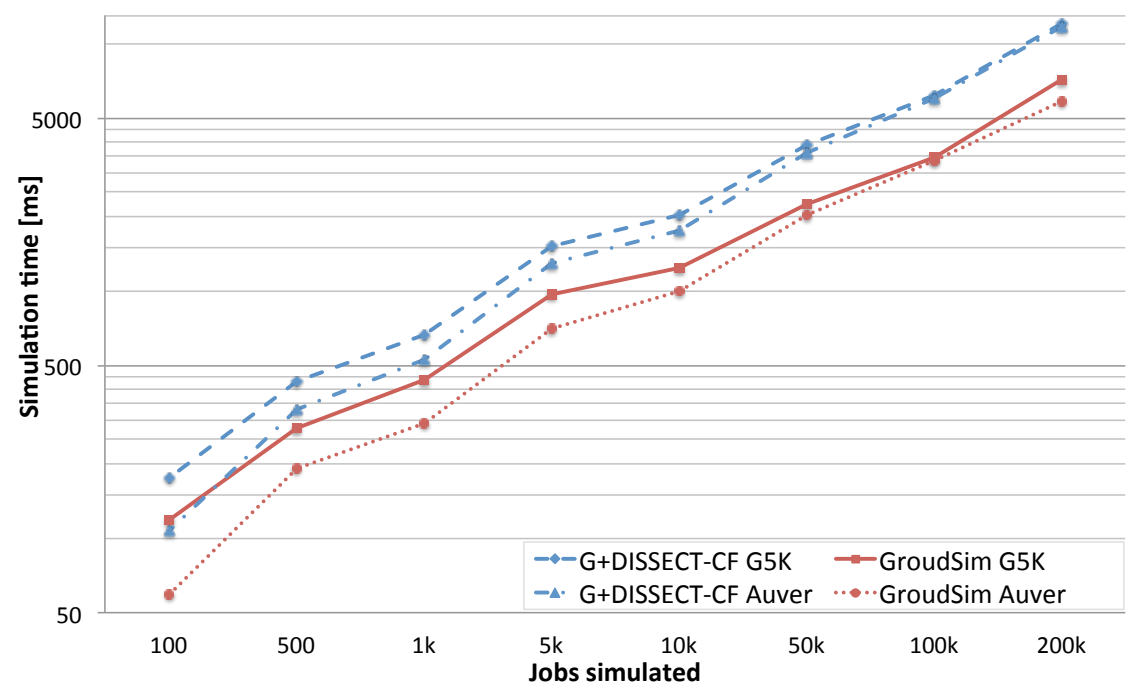

Figure 8. Scalability analysis of GroudSim and DISSECT-CF by simulating job-trace-files

traces represent real life scientific workloads. These traces were used because they are result in larger scale and more realistic simulations in contrast to the use of a single or repeated workflow executions otherwise possible through ASKALON.

Unfortunately, the GWA was focusing on the Grid workloads of the past. So it is not suitable on its own in a Cloud context. Despite there are several workload traces already available from the Cloud computing community, these traces are mostly limited to VM management operations and they rarely include VM-Task allocation information (which is an important aspect exploited in GroudSim). Also, these newer traces do not include enough information on user activities to evaluate the scalability penalties introduced in our integrated simulators. Thus, instead of using such Cloud specific workloads, we have extended the GWA traces to include VM management and VM-Task allocation as described in the following paragraph.

First of all, we interpreted job submissions in the GWA trace as VM requests. The number and the kind of VMs requested were determined by the number of processors required by the particular job to be run on the VMs (e.g., if there were VM types with 1, 2, 4, 8, 16, 32 and 64 cores, and the job required 1024 processors, then we have requested $16 \mathrm{VMs}$ with 64 processors). If the simulated Cloud could not serve the requested VMs at once, then we applied a simple policy that retried the VM requests after some of the previous jobs have completed. Finally, upon receiving notification from the simulator that the VMs for a specific job are ready then we have allocated the job to the newly prepared VMs in such parallel fragments that filled the VMs completely (e.g., with our previous example, the 1024 processor job was split to use all $16 \mathrm{VMs}$ in parallel).

During our evaluations with the GWA traces, in both the extended and the old simulators, we have prepared a Cloud infrastructure with a resource pool of 50 physical machines (each equipped with 64 cores, $128 \mathrm{GBs}$ of memory and 5TBs of disk). On this simulated infrastructure, we have executed the first $C$ (ranging from 100 to 200.000) jobs from the Auver Grid and from the Grid5000 traces in both the original and the extended simulators.

Figure 8 shows this performance analysis where one can observe that a small increase in execution time is notable for both traces but the scalability of GroudSim was not harmed by the integration of 


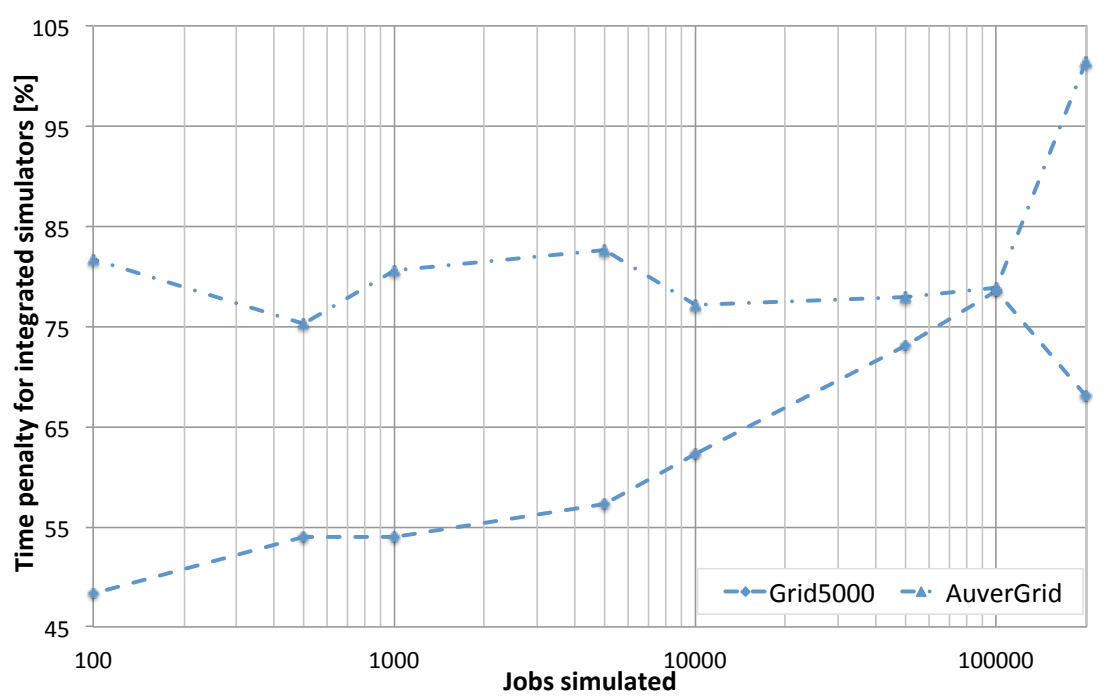

Figure 9. Relative performance drop of the extended simulator compared to its original form

DISSECT-CF. Comparing this overhead to the improved, more versatile and feature rich simulation, we concluded that this extended simulation time is still reasonable.

In terms of relative performance degradation compared to the original simulator, we have shown in Figure 9 that the Auver Grid trace simulation increased in execution time of $79 \%$ for most of the scenarios. Grid5000 was less affected by the integration and only had an additional execution time of $48 \%$ when simulating 100 tasks. For bigger simulations again a value around $80 \%$ was reached. The figure also shows that in smaller scale experiments, the relative performance of the simulators varies significantly (due to the internal behaviour of java VMs). On the other hand, after reaching around 10.000 simulated jobs, the overhead is ranging from $62 \%$ to $101 \%$ depending of the content of the traces. According to our measurements it never reaches more then $120 \%$ even with different traces than the ones we presented here.

Evaluation of basic VM creation performance. As the simulator is intended for evaluation of practical Cloud scenarios, we have investigated a typical use case in a Cloud setting when the virtual infrastructure for a SaaS provider needs to increase its size rapidly. In this case, the Cloud operator is requested to deliver a high amount of new VMs for the SaaS provider in a timely fashion. For this scenario we have set up a Cloud infrastructure within the simulators large enough to host the newly requested VMs of the SaaS provider. Next, we have prepared several traces (in the format of the GWA) that simultaneously requested $J \in\left\{10^{2}, 5 \cdot 10^{2}, 10^{3}, 5 \cdot 10^{3}, 10^{4}, 5 \cdot 10^{4}, 10^{5}, 2 \cdot 10^{5}\right\}$ single processor jobs (which were translated to small compute instance requests for our just prepared Cloud). These traces were used as the experiment setup for our experiment. After the simulators have executed all jobs in the traces (all of them in their separate small compute instances on the Cloud) we have both analysed how long did the simulation took to complete and how did the individual jobs performed according to the simulators. We used the second analysis to validate the simulators behavior (and found that the jobs in the new and in the old simulator completed with the same runtimes). Next, based on the simulation completion times we have drawn figure 10 . 


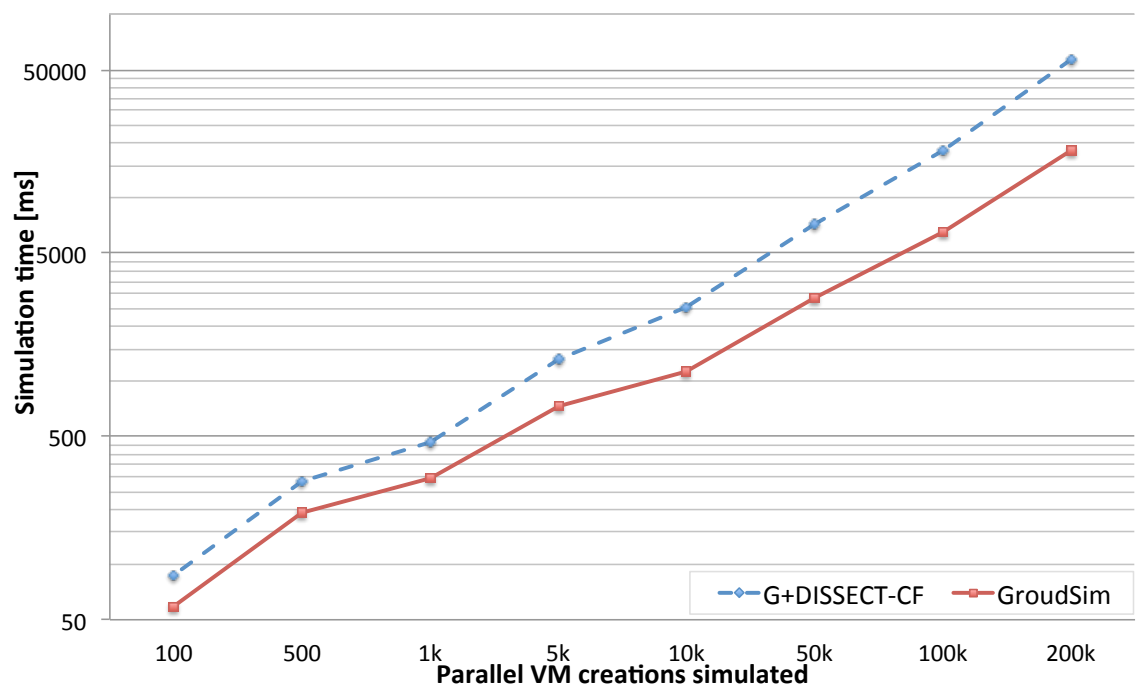

Figure 10. Scalability analysis of GroudSim and DISSECT-CF by requesting VMs in parallel

The figure shows that the bigger the parallelism, the bigger the scalability hit DISSECT-CF causes on GroudSim (e.g., the biggest performance degradation observed is over $3.1 \times$ ). This is caused by the extra simulation efforts made by DISSECT-CF (like VM startup simulation, network/disk transfers during VM image delivery, VM scheduling etc.). The higher performance degradations (i.e., for $J \geq 10^{4}$ the degradation is over $2.2 \times$ ) are mostly due to the current Cloud setup in the integrated simulator (where there is a single VM image store, thus the more VMs are requested in parallel the more likely this image store will become the bottleneck of the whole simulation). Fortunately, the performance degradation is less of a concern in smaller Cloud setups (e.g., Clouds with a few thousand compute nodes) or less parallel situations (e.g., request rates lower than a few hundred/s) which is mostly the target area of simulators like GroudSim. Amongst our future works in the integration, we plan to improve the automated Cloud creation process so it better matches the properties of the underlying DISSECT-CF simulation (e.g., by creating multiple VM image stores in a single Cloud), while we also plan to improve DISSECT-CF behavior under such level of parallelism.

Evaluation of basic networking behavior. For this experiment we have set up two hosts in the simulators and connected them with a gigabit ethernet connection. Then we have simultaneously started $T \in\{1,2,5,10,20,50,100,200,500,1000,2000,5000\}$ transfers on it. We have selected the numbers for simultaneous transfers to range between average (e.g., $T=1-10$ ) Cloud and more HPC like behaviors (e.g., $T>50$ ). For each network transfer the source and target hosts were selected in a round-robin fashion. And each transfer was simulated to be $B \in\left\{1,10^{2}, 10^{4}, 10^{6}, 10^{8}\right\}$ bytes in size. Similarly to the number of simultaneous transfers, the transfer size range was selected to allow the simulation of RPC (e.g., $B<10^{4}$ ) to transferring complete VM images $\left(B \geq 10^{8}\right)$. So an experiment setup has had two parameters: $\operatorname{setup}(T, B)$. For validation, we have run the experiments with all possible combinations of $T \times B$. Then we have compared the reported simulated transfer times for each transfer in both the new and the old simulators. We have found 


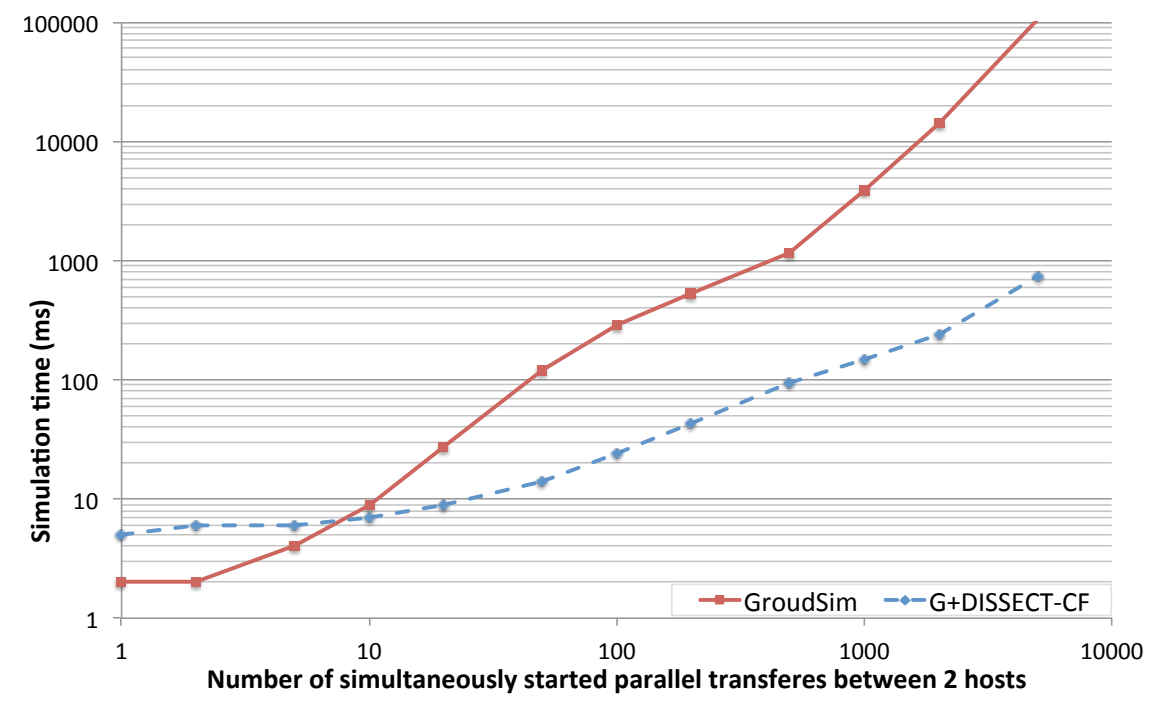

Figure 11. Scalability analysis of GroudSim and DISSECT-CF for network transfers

that the median difference between the two was negligible (smaller than the time resolution of the simulator). In contrast the average was a little bit over $31 \mathrm{~ms}(1.2 \%)$ stemmed from the different number representations in the two simulations.

Next, we have switched to the scaling experiment of the above network setup where we have run each experiment setup at least 10 times (according to equation (1)). The results are shown in Figure 11. Compared to the rest of the scalability measurements presented in this paper, this figure shows a little bit different behavior. First we can see that for transfers with little concurrency (i.e., $T \leq 5$ ), the performance of the combined simulators is lower just like in the other performance evaluation scenarios above, in fact the performance degradation caused by the integration is around $2.3 \times$. In contrast, the highly concurrent scenarios show that letting DISSECT-CF manage the whole network stack underneath GroudSim could bring significant advantages to its users. For the transfers in the HPC range $(T>50)$, the average performance improvement is $41 \times$. The performance improvements are mostly the result of the unified resource sharing foundation of DISSECT-CF which is especially tuned for highly parallel resource sharing scenarios like the one shown in the figure.

\subsection{New decision making opportunities in workflow research}

DISSECT-CF brings new features into the ASKALON ecosystem that will allow scientists, application developers and the ASKALON team to extend and improve their research in multiple areas and directions. In the following listing, we introduce those research areas that are newly available in the extended ASKALON. In Section 6 we will discuss additional research areas planned to be covered in the future.

Network usage. GroudSim was developed with very little focus on network functionality as back then the focus of all workflows used in ASKALON was on the computational part. In these workflows file dependencies took only a marginal amount of data that have had to be transferred between resources. With integration of DISSECT-CF the network model of 
GroudSim was replaced with a more accurate one that allows more precise simulation of data intensive applications. As a result, scheduling techniques that consider data movement can exploit the more accurate file transfer predictions for such applications and can improve workflow runtimes more precisely.

Data centre configurations. DISSECT-CF allows to specify the characteristics of data centres in an easily exchangeable configuration file. Utilising this mechanism it can be evaluated what kind of data centre might be best fitting for a specific kind of workflow application and also make simulations based on existing data centres configurations. This was not possible with GroudSim as the hardware model of data centres was not existing. We aim at determining the influence of data centre configuration on workflow applications and their schedule with a series of experiments in the near future that will show how important it is do simulate Clouds not only as black boxes but that internal hardware specifications have influence on the overall performance.

With the ongoing development of DISSECT-CF the supported research directions will be further extended. ASKALON users and developers will directly benefit from each new feature developed and will allow scientists to develop new methods, algorithms and solutions to Cloud and workflow management related problems.

\subsection{Workflow simulation details}

In this section we show how ASKALON can be used to simulate the execution of scientific workflow applications on IaaS Cloud resources. Taking real execution data (to better predict the runtime of the simulated activities) we preformed a set of experiments to show how easy it is to simulate runs that normally would take weeks to execute and would cost thousands of dollars for the used Cloud instances.

The integration of DISSECT-CF into GroudSim also provided access to the new features added and higher precision to the ASKALON environment. In ASKALON, workflows can be executed in real systems as well as in simulated environments allowing the evaluation of new execution scenarios without needing to actually pay for the underlying simulated infrastructure. Currently, the simulated workflows can run on dynamic Cloud resources leased from a IaaS provider and more static Grid resources similar to academic infrastructures as the Austrian Grid. Workflow execution is a complex topic where multiple problems are known to be NP-complete like scheduling of tasks to a heterogeneous set of resources. To allow faster evaluation of new workflow execution techniques and optimizations, simulation is widely used because it allows important observations like resource utilisation, cost, energy consumption to be collected.

Figure 12 shows a screenshot of the main ASKALON graphical user interface used for developing workflows and submitting them for execution (the figure actually shows the Wien2k workflow application to be explained in Section 5.4). Additional to command line tools this is the main tool to execute workflows.In the left side tree a list of available applications is shown to the user. Those can be drag and dropped in the main area on the right side. Activities are connected with control flow edges from the initial node towards the final node of the workflow. The bottom part shows on the left the services the GUI is currently connected to and some log messages of the ongoing execution on the right. 


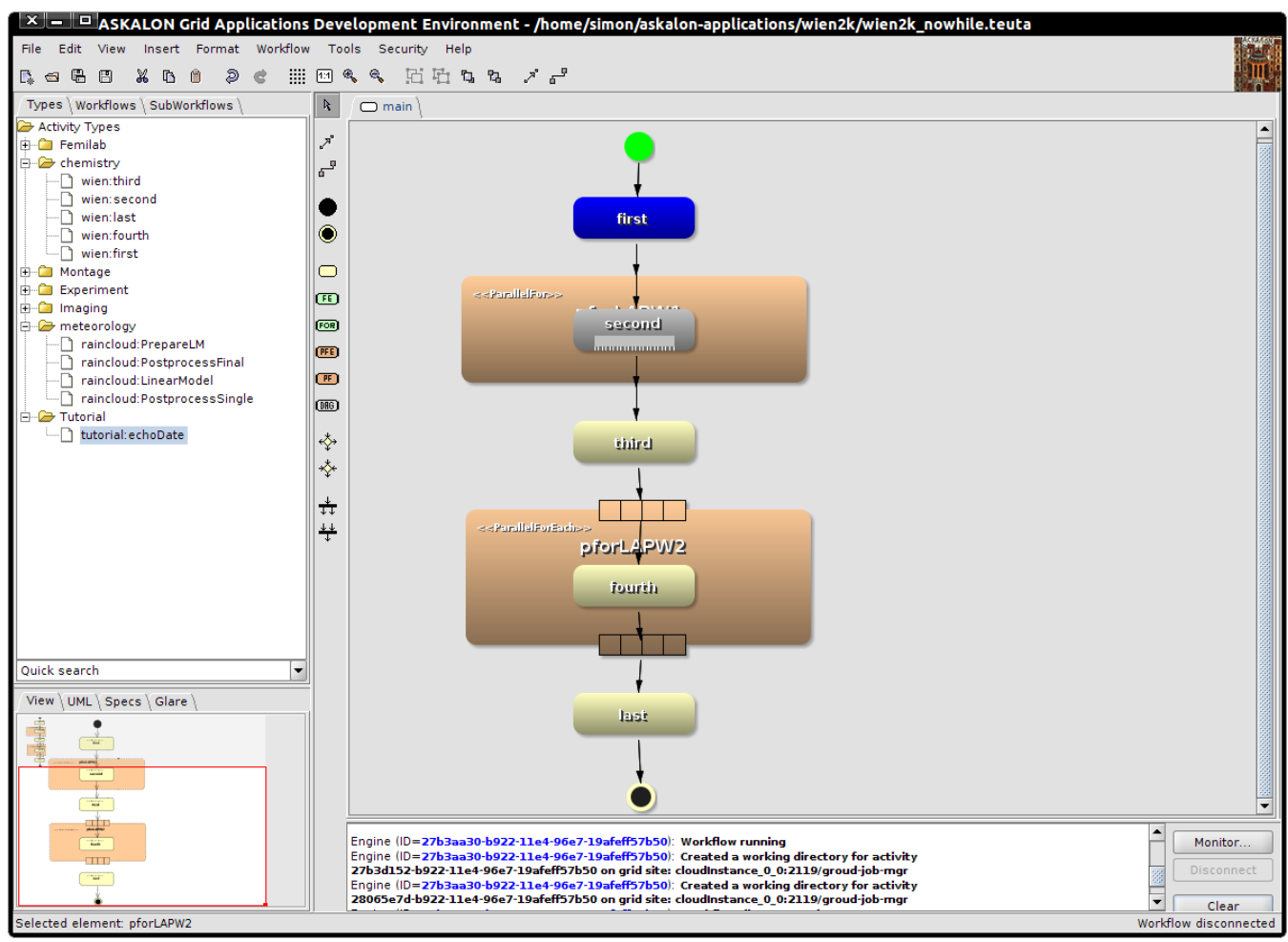

Figure 12. Workflow creation and execution GUI showing Wien2k [27]

In most environments scientists first craft their ideas on paper, then implement them in a simulator and if evaluated to be an improvement over existing technologies it might get implemented for a real system. In the case of ASKALON step 2 and 3 are combined, as the initial implementation for simulated evaluation can already be done in the system the algorithm is designed for. Switching from simulation to execution only requires the change of a flag for the execution environment and all resource requests, file transfers or job submissions are either sent to GroudSim or to the real environment.

Figure 13 shows the performance and monitoring tool included in ASKALON. It can be used to visualise the execution in a gannt-chart like fashion and allows control of what is shown: all activities, activities per host, file transfers, Cloud machines, preparation jobs, tear-down jobs and more. On the left a tree representation of the unrolled workflow is given, allowing to show only the selected sub parts of the workflow by selecting the responsible parts in the tree. ASKALON internal stores all the events happening touring a execution in a database. This events are then used to visualise the workflow execution in this tool. The tool can be used while workflows are running using a periodical refresh or after workflow execution finished.

The GroudSim enabled execution service, based on a globus web services resource framework, additionally provides the user with a user interface that allows to simply create, store or save the infrastructure configurations he/she wants to be used within the simulation (Figure 14 shows this GUI). Users can even set up infrastructures with a mixture of Cloud and Grid resources as ASKALON supports execution on such hybrid hardware environments. Simple statistics (like the number of simulated jobs or file transfers) are visualised for the user and tools are available to start 


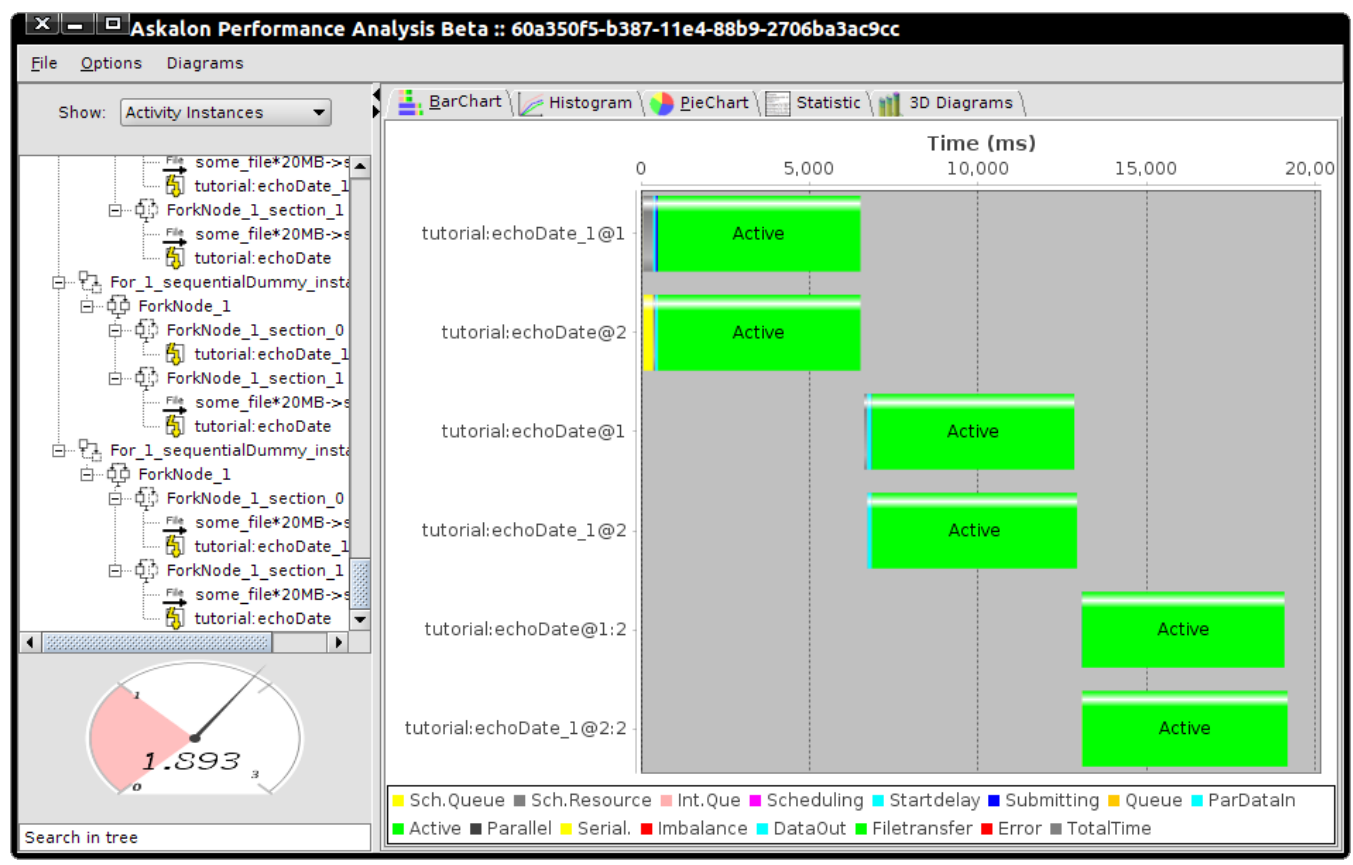

Figure 13. Workflow monitoring and performance tool from the ASKALON suite

or stop Cloud instances, define resource requirements of tasks and give custom file sizes if needed. All settings accessible in the GUI can also be loaded using configuration files to allow massive simulation runs that have no need for user interaction.

There are additional tools to manage and monitor the simulation, because despite the standard ASKALON monitoring tools are also working for simulated workflow executions, their recorded timestamps for the events could easily be in the future. For example, for a simulated workflow execution that finished in a few seconds but in real life it would have taken several hours of runtime, the ASKALON monitoring database would show completion times for the workflow's jobs in the future for a while.

In simulated experiments, use cases, which are often hard to (re)produce, like failing resources, network connections going down, spot instances getting terminated and similar, are especially important. Those events might occur any time during a workflow execution and therefore a workflow system should be able to deal with such faults. As physical access to networks are not always granted it might not be that easy to physically take 1 region of a Cloud provider (i.e. Amazon EC2) offline without access to network configurations beyond one's control.

\subsection{Wien $2 k$ Workflow experiments}

In this subsection, we present experiments done simulating a scientific workflow application called Wien $2 \mathrm{k}$ and we show how to do large scale simulations using the presented software stack.

Wien2k [27] is a material science workflow for performing electronic structure calculations of solids using density functional theory based on the full-potential (linearized) augmented plane-wave ((L)APW) and local orbital (lo) method. The Wien2k workflow contains two parallel sections of size $x$, with sequential synchronization activities in between. Figure 15 shows the scientific parts of the workflow while in Figure 12 the workflow modeled from those parts can be seen. Each activity in 


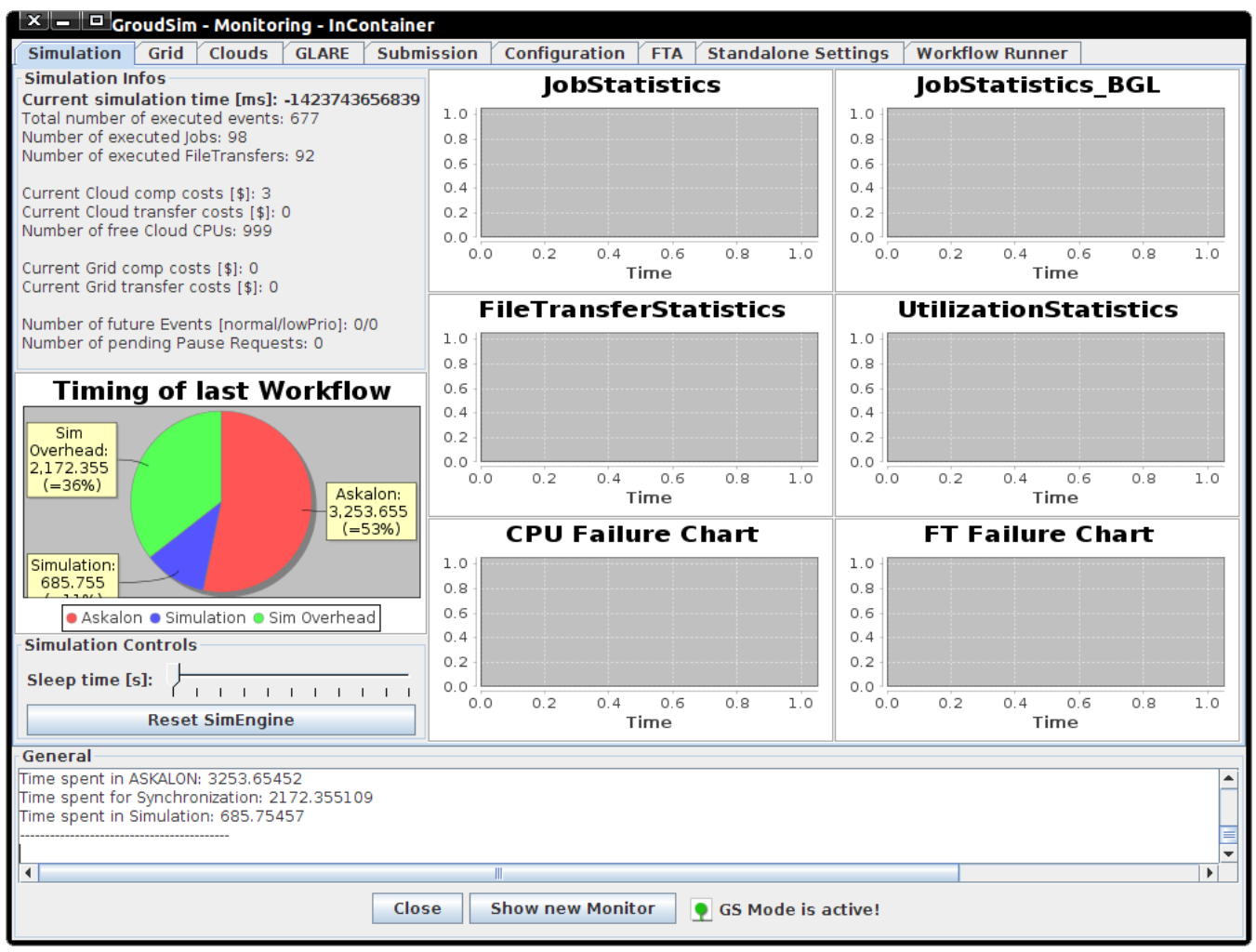

Figure 14. GUI to manipulate the GroudSim settings in ASKALONS simulation mode

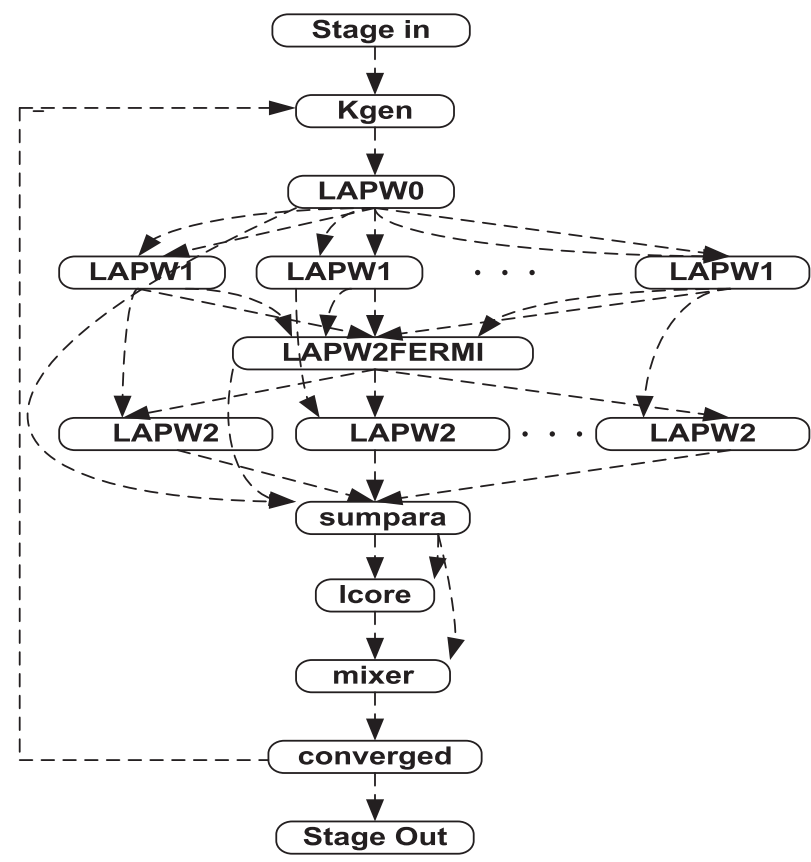

Figure 15. The scientific workflow application Wien2k.

the screenshot represents one or multiple tasks from the aplication i.e. first consists of Kgen and LAPWO and last of sumpara till Stage Out.. The other three activities are mapped to one task each: 


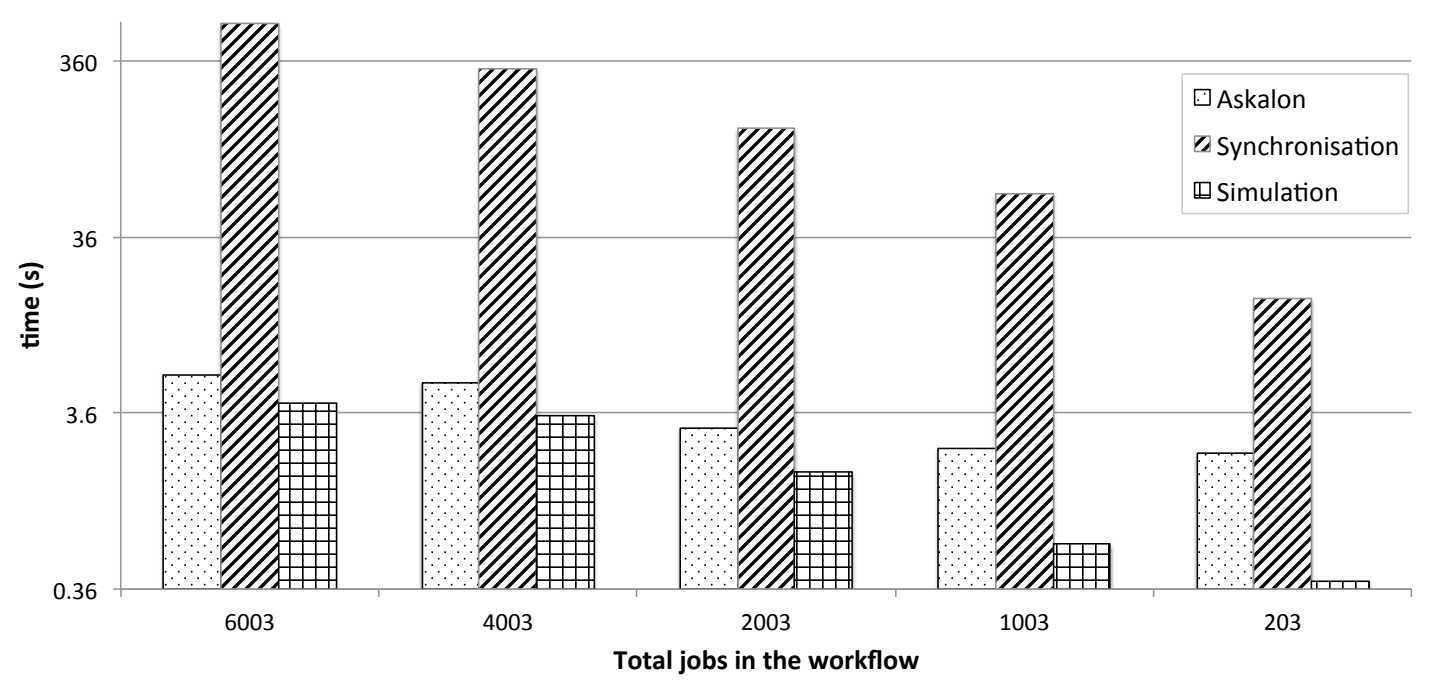

Figure 16. Simulation times for Wien2k workflow runs with $x=100,500,1000,2000,3000$.

second is $L A P W 1$, third is LAPW2FERMI and forth is LAPW2 The total number of activities in a Wien2k workflow is: $N_{\text {wien } 2 k}=2 \cdot x+3$. For simplicity the activities of the workflow are named: first, second (parallel part in a parallel for loop), third, fourth (parallel part in a parallel for each section) and last.

In the simulated case, the application itself is not executed and no real resources are used expect of the machines running the integrated simulators and the ASKALON execution engine. This experiment shows the scalability of the workflow system and the overheads introduced by the synchronication between EE2 and GroudSim using the GAB component.

Figure 16 shows the execution times of multiple workflow simulations executed on a desktop computer with a Intel i7-2600k CPU and 8 gigabytes of memory running Ubuntu. All components of the simulations were executed on the same host to represent a standard use case where developers run simulations directly on their workstation. The chart shows that simulating a workflow with $100 \mathrm{k}$-points (resulting in 203 tasks and 1418 file transfers) takes in average 18,7 seconds while workflows with 3000 k-points (and 6003 tasks and 42018 file transfers) take in average 10 minutes. This quite poor performance compared to the quite more impressive GroudSim stand alone performance can be explained with the multiple synchronisation points between the simulator and the workflow system. As the chart shows, the simulation's execution time are not significantly influencing the simulated runtime of the ASKALON workflow (because the synchronisation of the ASKALON execution engine and the GAB web services are the biggest bottleneck in our experiments - e.g., for every simulated job the completion is propagated back to the ASKALON GUI which was not an issue in real infrastructures with long runtimes but now it became a serious limitation). Therefore, within the context of ASKALON, we receive the precision and detail improvements of DISSECT-CF practically with no apparent costs for the user.

Taking into account the times only consumed by the simulator then the time for the smallest run with $100 \mathrm{k}$-points took only 0,4 seconds. The time spend in ASKALON is hard to identify as there is a high overlap with synchronisation times. ASKALONs execution engine is heavily thread based and only a few of them get blocked in the synchronisation parts and have to wait for 
Table I. Real world execution cost and time of Wien2k runs from simulated examples.

\begin{tabular}{l|c|c|c|c}
\hline k-points & tasks & file transfers & cost [\$] & runtime $[\mathrm{h}]$ \\
\hline 100 & 203 & 1418 & 53,46 & 127,3 \\
500 & 1003 & 7018 & 320,14 & 623,77 \\
1000 & 2003 & 14018 & 637,7 & 1235,1 \\
2000 & 4003 & 28018 & 1273,0 & 2482,5 \\
3000 & 6003 & 42018 & 1908,2 & 3692,8 \\
\hline
\end{tabular}

simulator reactions. 13-16 seconds are covering this synchronisation part and about 1-3 seconds can be addressed to ASKALON only. To improve this performance we plan to work on the GAB to allow bundled event transfer between ASKALON to the simulator to reduce the amounts of changes between simulation and real-time behaviour. Once the GAB allows bundled event transfer the performance degradations in the integrated GroudSim will be more apparent but we expect that the synchronisation time will still dominate the executions, thus in the future even more features could be added to GroudSim without losing user side performance. Again the integration of DISSECT-CF does show very little influence on the overall performance and does not become the bottleneck of the simulation system.

We collected the cost for the Cloud resources we used within those simlated executions and present those results in table I. For the cost calculation in the simulator we used the informations provided from Amazon EC2: c3.2xlarge with 8 cores and a total of 28 ECU, 15 gigabyte of memory and 2 x 80 SSD hard drives (which are not simulated by the current system) for $\$ 0.420$ per Hour based on the US East regions cost.

Table I shows that it would take 22 weeks an cost nearly $\$ 2.000,0$ to run the biggest workflow we simulated on an environment like Amazon EC2. Even though execution time might be decreased with more resources at in parallel but still the cost would slightly increase with higher resource usage. Comparing this to the simulation time of only 10 minutes and no cost except of the power for the desktop computer let us conclude, that simulation is a important tool for workflow developers. When new optimizations are evaluated and multiple workflow executions are needed to validate the approach, simulation is often the only feasible solution.

\section{CONCLUSION AND FUTURE WORKS}

When evaluating scientific research, simulation tools are invaluable alternatives to real-world environments. For example in the field of scientific workflow management systems, simulators enable faster, more versatile, deterministic, and reproducible experimentation, including situations not easily reproducible in real-life. Despite their importance, current Cloud workflow simulators lack sufficient support with respect to the underlying virtualised infrastructure, including energyawareness that is highly demanded in today's data centres. To address this gap, we presented in this paper the integration of a stand-alone DIScrete event baSed Energy Consumption simulaTor for Clouds and Federations (DISSECT-CF) with a mature real-world Cloud workflow management system called ASKALON and its underlying Grid/Cloud simulation environment called GroudSim. We discussed the challenges that appeared as the result of the originally incompatible APIs 
and functionalities of ASKALON, GroudSim and DISSECT-CF, and presented the required reengineering and adjustments in three main areas: $(i)$ event system, including event types, firing mechanisms and clock maintenance techniques, (ii) Cloud representation at the level of data centre, VM and job management, and finally (iii) network construction, utilisation, sharing and organisation.

Our experimental evaluation, conducted on an over 3000 core simulated Cloud infrastructure, demonstrated an improved behaviour of the ASKALON system regarding networking, energy metering, VM instantiation and CPU sharing accuracy while the performance of the integrated simulatiors never dropped below half of the original GroudSim based simulations. We concluded that despite the improved functionality, the scalability of the simulator did not drop and was in alignment with our past results where we have shown the scaling issues in relation with simulators [7] and evaluated the performance of simulating large skale workflow experiments with the ASKALON environment. We identified optimization possibilities in the following fields: $(i)$ resource utilisation improvements, $(i i)$ power consumption optimisations for workflows and Cloud providers, (iii) network aware workflow scheduling, and (iv) optimising workflow executions depending on data centre configurations.

Future work will target improvements in the GroudSim-ASKALON bridge allowing more information to be shared with the simulators regarding the executed workflows and also allowing ASKALON environment to gather more details about the simulated infrastructures. We will also focus on reducing the performance overheads caused by the duplication of some functionalities in the system (e.g., eventing) allowing GroudSim to concentrate more on the user side behaviour of Clouds and Grids. Finally, we plan to introduce dynamic pricing models to GroudSim by relying on DISSECT-CF's resource utilisation and energy consumption related reports. For the following research directions, that we target, made possible by this additional feature forwarding from DISSECT-CF to the workflow system, we want to give a short overview of the upcoming research areas:

Power consumption. Green IT is getting more important, as power consumption and resulting CO2 emissions are becoming widely known issues to the general public. Workflow schedulers can offer benefits for customers by improving scheduling and resource management through the use of DISSECT-CF provided measurements about the power draw of physical resources. The collected power measurements then allow the optimisation of workflow execution considering not only cost and time but also energy consumption. Although, contemporary Cloud systems lack this metering functionality, enabling research work on the area will increase demands towards providers and prepare novel workflow management systems for times when such features become available from commercial or academic Clouds.

Cloud providers pay special attention to energy consumption reduction as it can directly reduce data centre operating costs. These cost reductions then can either give a competitive pricing advantage to the provider or increase its margins allowing more funds for its activities. Research in the area of VM placement is therefore not only interesting for users but also providers. Data centres could advertise that they apply environment friendly policies and users that want to support power saving would get attracted by such providers similar to renewable 
energy producers (which manage to sell their energy in most cases even more expensive then regular providers).

Resource usage. The new functionality of DISSECT-CF allows the identification of different physical machines for the instantiated VMs. This does not only allow to invent new methods for IaaS internal resource mapping but also in combination with workflow execution can improve the resource utilisation. In most cases, Cloud providers are seen as black boxes where the mapping of VMs to physical resources can only be guessed. With the integration of DISSECT-CF into GroudSim, new possibilities were opened up in ASKALON schedulers and resource managers. Knowing which instances share a physical machine can be used by the scheduler to map tasks with high data dependencies on instances that are close to each other resulting in reduced data transfer times.

New research directions can also be utilised within the IaaS provider. It is now possible to investigate different policies for physical - VM mapping and their influence on performance, power consumption, utilisation and fairness. To investigate those features, internal IaaS scheduling mechanisms need to be changed which would not have been possible in GroudSim before the integration of DISSECT-CF. GroudSim had anonymous resource pools of cores only and did not understand the concept of physical machines.

Additional research in the IaaS area is planed within the H2020 project named EntICE [28] that will focus on multi parameter optimization of Cloud image repositories, including power as an important parameter.

\section{ACKNOWLEDGEMENT}

The work presented in this paper has been partially supported by the Austrian Science fund project TRP 237-N23, by the EU under the COST programme Action IC1305, 'Network for Sustainable Ultrascale Computing (NESUS) and by the EU H20 project ENTICE: dEcentralized repositories for traNsparent and efficienT vIrtual maChine opErations 644179.

\section{REFERENCES}

1. Taylor IJ, Deelman E, Gannon DB, Shields M. Scientific Workflows for Grids. Workflows for e-Science, Springer Verlag, 2007.

2. Armbrust M, Fox A, Griffith R, Joseph AD, Katz R, Konwinski A, Lee G, Patterson D, Rabkin A, Stoica I, et al. A view of cloud computing. Commun. ACM Apr 2010; 53(4):50-58, doi:10.1145/1721654.1721672. URL http://doi.acm.org/10.1145/1721654.1721672.

3. Plankensteiner K, Prodan R, Janetschek M, Montagnat J, Rogers D, Harvey I, Taylor I, Balaskó Á, Kacsuk P. Finegrain interoperability of scientific workflows in distributed computing infrastructures. Journal of Grid Computing 2013; 11(3):429-455, doi:10.1007/s10723-013-9261-8.

4. Ahmed A, Sabyasachi A. Cloud computing simulators: A detailed survey and future direction. Advance Computing Conference (IACC), 2014 IEEE International, 2014; 866-872, doi:10.1109/IAdCC.2014.6779436.

5. Sakellari G, Loukas G. A survey of mathematical models, simulation approaches and testbeds used for research in cloud computing. Simulation Modelling Practice and Theory 2013; 39:92-103.

6. Calheiros RN, Ranjan R, Beloglazov A, De Rose CAF, Buyya R. Cloudsim: a toolkit for modeling and simulation of cloud computing environments and evaluation of resource provisioning algorithms. Software: Practice and Experience 2011; 41(1):23-50, doi:10.1002/spe.995. URL http: / / dx. doi .org/10.1002/spe. 995. 
7. Ostermann S, Plankensteiner K, Prodan R. Using a new event-based simulation framework for investigating resource provisioning in clouds. Scientific Programming 2011; 19(2):161-178.

8. Nuñez A, Vázquez-Poletti JL, Caminero AC, Carretero J, Llorente IM. Design of a new cloud computing simulation platform. Computational Science and Its Applications-ICCSA 2011. Springer, 2011; 582-593.

9. Ullman JD. Np-complete scheduling problems. J. Comput. Syst. Sci. Jun 1975; 10(3):384-393, doi:10.1016/ S0022-0000(75)80008-0. URL http://dx.doi.org/10.1016/s0022-0000 (75)80008-0.

10. Ostermann S, Plankensteiner K, Prodan R, Fahringer T, Iosup A. Workflow monitoring and analysis tool for ASKALON. Grid and Services Evolution, Barcelona, Spain, 2008; 73-86.

11. Kecskemeti G. Dissect-cf: a simulator to foster energy-aware scheduling in infrastructure clouds. Submitted to: Simulation Modelling Practice and Theory 2014; .

12. Rogers D, Harvey I, Huu TT, Evans K, Glatard T, Kallel I, Taylor I, Montagnat J, Jones A, Harrison A. Bundle and pool architecture for multi-language, robust, scalable workflow executions Sep 2013; 11(3):457-480, doi: http://dx.doi.org/10.1007/s10723-013-9267-2. URL http:// link.springer.com/article/10.1007/ s10723-013-9267-2.

13. Buyya R, Murshed M. Gridsim: A toolkit for the modeling and simulation of distributed resource management and scheduling for grid computing. Concurrency and computation: practice and experience 2002; 14(13-15):11751220.

14. Murugesan S. Harnessing green it: Principles and practices. IT Professional Jan 2008; 10(1):24-33, doi:10.1109/ MITP.2008.10. URL http://dx.doi.org/10.1109/MITP.2008.10.

15. Chen W, Deelman E. Workflowsim: A toolkit for simulating scientific workflows in distributed environments. $E$ Science (e-Science), 2012 IEEE 8th International Conference on, IEEE, 2012; 1-8.

16. Casanova H, Giersch A, Legrand A, Quinson M, Suter F. Versatile, scalable, and accurate simulation of distributed applications and platforms. Journal of Parallel and Distributed Computing 2014; 74(10):2899 - 2917, doi:http: //dx.doi.org/10.1016/j.jpdc.2014.06.008. URL http://www. sciencedirect.com/science/article/ pii/S0743731514001105.

17. Ostermann S, Plankensteiner K, Bodner D, Kraler G, Prodan R. Integration of an event-based simulation framework into a scientific workflow execution environment for grids and clouds. Towards a Service-Based Internet, Lecture Notes in Computer Science, vol. 6994. Springer: Poznan, Poland, 2011; 1-13, doi:10.1007/978-3-642-24755-2_1.

18. Lee K, Paton NW, Sakellariou R, Deelman E, Fernandes AAA, Mehta G. Adaptive workflow processing and execution in pegasus. Workshops at the Grid and Pervasive Computing Conference, GPC 2008, Kunming, China, May 25-28, 2008, IEEE Computer Society, 2008; 99-106, doi:10.1109/GPC.WORKSHOPS.2008.30. URL http: //doi. ieeecomputersociety.org/10.1109/GPC.WORKSHOP S.2008.30.

19. Wolstencroft K, Haines R, Fellows D, Williams AR, Withers D, Owen S, Soiland-Reyes S, Dunlop I, Nenadic A, Fisher P, et al.. The taverna workflow suite: designing and executing workflows of web services on the desktop, web or in the cloud. Nucleic Acids Research 2013; 41(Webserver-Issue):557-561, doi:10.1093/nar/gkt328. URL http://dx.doi.org/10.1093/nar/gkt328.

20. Farkas Z, Kacsuk P. P-GRADE portal: A generic workflow system to support user communities. Future Generation Comp. Syst. 2011; 27(5):454-465, doi:10.1016/j.future.2010.12.001. URL http://dx. doi .org/10.1016/ j.future.2010.12.001.

21. von Laszewski G, Foster IT, Gawor J. CoG kits: a bridge between commodity distributed computing and highperformance Grids. Java Grande Conference, ACM Press, 2000; 97-106.

22. Barrionuevo JJD, Fard HM, Prodan R. MOHEFT: A multi-objective list-based method for workflow scheduling. 4th IEEE International Conference on Cloud Computing Technology and Science Proceedings, CloudCom 2012, Taipei, Taiwan, December 3-6, 2012, IEEE Computer Society, 2012; 185-192, doi:10.1109/CloudCom.2012.6427573. URL http://dx.doi.org/10.1109/CloudCom.2012.6427573.

23. Wieczorek M, Prodan R, Fahringer T. Scheduling of scientific workflows in the ASKALON grid environment. SIGMOD Record 2005; 34(3):56-62, doi:10.1145/1084805.1084816. URL http://doi.acm.org/10. $1145 / 1084805.1084816$.

24. Braun TD, Siegel HJ, Beck N, Bölöni L, Maheswaran M, Reuther AI, Robertson JP, Theys MD, Yao B, Hensgen DA, et al.. A comparison of eleven static heuristics for mapping a class of independent tasks onto heterogeneous distributed computing systems. J. Parallel Distrib. Comput. 2001; 61(6):810-837, doi:10.1006/jpdc.2000.1714. URL http://dx.doi.org/10.1006/jpdc.2000.1714.

25. Sulistio A, Cibej U, Venugopal S, Robic B, Buyya R. A toolkit for modelling and simulating data Grids: an extension to GridSim. Concurrency and Computation: Practice and Experience 2008; 20(13):1591-1609.

26. Iosup A, Li H, Jan M, Anoep S, Dumitrescu C, Wolters L, Epema DHJ. The grid workloads archive. Future Generation Comp. Syst. 2008; 24(7):672-686, doi:10.1016/j.future.2008.02.003. URL http: / / dx. doi .org/ $10.1016 / j$. future.2008.02.003. 
27. Schwarz K, Blaha P, Madsen G. Electronic structure calculations of solids using the wien $2 \mathrm{k}$ package for material sciences. Computer Physics Communications 2002; 147(1):71-76.

28. February 2015. URL http: / / www. entice-project.eu. 